\title{
On "GSI Oscillations" as Interference of Two Closely Spaced Ground Mass Eigenstates of H-Like Mother Ions
}

\author{
M. Faber, ${ }^{1}$ A. N. Ivanov, ${ }^{1}$ P. Kienle, ${ }^{2,3}$ E. L. Kryshen, ${ }^{4}$ M. Pitschmann, ${ }^{1}$ and N. I. Troitskaya ${ }^{5}$ \\ ${ }^{1}$ Atominstitut der Österreichischen Universitäten, Technische Universität Wien, Wiedner Hauptstrasse 8-10, 1040 Wien, Austria \\ ${ }^{2}$ Stefan Meyer Institut für subatomare Physik, Österreichische Akademie der Wissenschaften, Boltzmanngasse 3, 1090 Wien, Austria \\ ${ }^{3}$ Excellence Cluster Universe, Technische Universität München, 85748 Garching, Germany \\ ${ }^{4}$ St. Petersburg Nuclear Physics Institute, Orlova Roscha 1, Gatchina 188300, Russia \\ ${ }^{5}$ State Polytechnic University of St. Petersburg, Polytechnicheskaya 29, St. Petersburg 195251, Russia
}

Correspondence should be addressed to A. N. Ivanov, ivanov@kph.tuwien.ac.at

Received 26 June 2009; Accepted 4 August 2009

Recommended by Peter Blunden

We analyse the hypothesis that the "GSI oscillations" of the K-shell electron capture decay (EC) rates of the H-like heavy ions are caused by quantum beats from a coherent state of two closely spaced ground mass-eigenstates $\left|m^{\prime}\right\rangle$ and $\left|m^{\prime \prime}\right\rangle$ of decaying H-like heavy ions. We apply this mechanism to the calculation of the $\beta^{+}$-decay rates of the H-like heavy ions and discuss the dynamics of the production of the H-like heavy ions with two closely spaced ground mass-eigenstates at GSI experiments. We show that such a mechanism cannot describe simultaneously the experimental data on both the EC-decay and $\beta^{+}$-decay rates of the $\mathrm{H}$-like heavy ions, measured at GSI.

Copyright (C) 2009 M. Faber et al. This is an open access article distributed under the Creative Commons Attribution License, which permits unrestricted use, distribution, and reproduction in any medium, provided the original work is properly cited.

\section{Introduction}

Recently Litvinov et al. [1] have observed that the K-shell electron capture (EC) decay rates of $\mathrm{H}$-like ${ }^{140} \mathrm{Pr}^{58+}$ and ${ }^{142} \mathrm{Pm}^{60+}$ ions

$$
\begin{aligned}
& { }^{140} \mathrm{Pr}^{58+} \longrightarrow{ }^{140} \mathrm{Ce}^{58+}+v_{e} \\
& { }^{142} \mathrm{Pm}^{60+} \longrightarrow{ }^{142} \mathrm{Nd}^{60+}+v_{e}
\end{aligned}
$$

have an unexpected periodic time modulation of exponential decay curves. The rates of the number $N_{d}^{\mathrm{EC}}$ of daughter ions ${ }^{140} \mathrm{Ce}^{58+}$ and ${ }^{142} \mathrm{Nd}^{60+}$

$$
\frac{d N_{d}^{\mathrm{EC}}(t)}{d t}=\lambda_{\mathrm{EC}}(t) N_{m}(t),
$$

where $N_{m}(t)$ is the number of the $\mathrm{H}$-like mother ions ${ }^{140} \operatorname{Pr}^{58+}$ or ${ }^{142} \mathrm{Pm}^{60+}[1]$ and $\lambda_{\mathrm{EC}}^{(\mathrm{H})}(t)$ is the EC-decay rate, are periodic functions, caused by a periodic time dependence of the ECdecay rates

$$
\lambda_{\mathrm{EC}}(t)=\lambda_{\mathrm{EC}}\left(1+a_{\mathrm{EC}} \cos \left(\omega_{\mathrm{EC}} t+\phi_{\mathrm{EC}}\right)\right)
$$

with a period $T_{\mathrm{EC}}=2 \pi / \omega_{\mathrm{EC}} \simeq 7$ seconds, an amplitude $a_{\mathrm{EC}} \simeq 0.20$, and a phase $\phi_{\mathrm{EC}}$.

In the articles [2-4] we have proposed an explanation of the periodic time dependence of the EC-decay rates as an interference of two neutrino mass eigenstates $v_{1}$ and $v_{2}$ with masses $m_{1}$ and $m_{2}$, respectively. The period $T_{\mathrm{EC}}$ of the time dependence has been related to the difference $\Delta m_{21}^{2}=$ $m_{2}^{2}-m_{1}^{2}$ of the squared neutrino masses $m_{2}$ and $m_{1}$ as follows:

$$
\omega_{\mathrm{EC}}=\frac{2 \pi}{T_{\mathrm{EC}}}=\frac{\Delta m_{21}^{2}}{2 \gamma M_{m}},
$$

where $M_{m}$ is the mass of the mother ion and $\gamma=1.43$ is a Lorentz factor [1]. In a subsequent analysis we also showed that the $\beta^{+}$branches of the decaying $\mathrm{H}$-like heavy ions do not show time modulation, because of the broad energy spectrum of the neutrinos in the corresponding three-body decays and proposed a test of such a behaviour [4].

According to atomic quantum beat experiments [5-7], the explanation of the "GSI oscillations," proposed in [2], bears similarity with quantum beats of atomic transitions, when an excited atomic eigenstate decays into a coherent state of two (or several) lower lying atomic eigenstates. 
In the case of the EC-decay one deals with a transition from the initial state $|m\rangle$ to the final state $\left|d v_{e}\right\rangle$, where the electron neutrino is a coherent superposition of two neutrino mass eigenstates with the energy difference equal to $\omega_{21}=$ $\Delta m_{21}^{2} / 2 M_{m}$ related to $\omega_{\mathrm{EC}}$ as $\omega_{\mathrm{EC}}=\omega_{21} / \gamma$.

Another mechanism of the "GSI oscillations" has been proposed by Giunti [8] and Kienert et al. [9]. The authors $[8,9]$ assume the existence of two closely spaced ground mass eigenstates of the mother of the H-like heavy ion in the initial state of the EC-decay and describe the initial state of the mother ion by the coherent superposition

$$
|m\rangle=\cos \theta\left|m^{\prime}\right\rangle+\sin \theta\left|m^{\prime \prime}\right\rangle
$$

of the wave functions of two mass eigenstates $\left|m^{\prime}\right\rangle$ and $\left|m^{\prime \prime}\right\rangle$ with masses $M_{m^{\prime}}$ and $M_{m^{\prime \prime}}$, respectively, and mass splitting of order $\Delta E_{m^{\prime} m^{\prime \prime}}=M_{m^{\prime}}-M_{m^{\prime \prime}} \sim 10^{-15} \mathrm{eV} ; \theta$ is a mixing angle.

Unlike our analysis [2-4], the authors [8, 9] draw an analogy of the "GSI oscillations" with quantum beats of atomic transitions [7], when an atom, excited into a state of a coherent superposition of two closely spaced energy eigenstates, decays into a lower lying energy eigenstate. According to [7], the intensity of radiation, caused by a transition from such a coherent state into a lower energy eigenstate, has a periodic time dependent term with a period inversely proportional to the energy-difference $\Delta E_{m^{\prime} m^{\prime \prime}}$ between two closely spaced energy eigenstates.

In this paper we apply the mechanism, proposed in $[8,9]$, to the analysis of the time modulation of the $\beta^{+}$-decay rates of the H-like heavy ions. We analyse also the dynamics of the production of the $\mathrm{H}$-like heavy ions with two closely spaced ground mass eigenstates at GSI experiments.

The mass splitting $\Delta E_{m^{\prime} m^{\prime \prime}}=M_{m^{\prime}}-M_{m^{\prime \prime}} \sim 10^{-15} \mathrm{eV}$ can be attributed either to the nucleus or to the energy level of the bound electron of the H-like mother ion. If the mass splitting is related to the energy level of the bound electron, one can show that in this case the coherent state $|m\rangle$, normalised to unity, reduces to the wave function of the unperturbed state of the H-like mother ion with a time dependent phase, which leads to no time modulation for the EC-decay rate of the H-like mother ion. Thus, we analyse below only the case, when the mass splitting is related to the nucleus of the $\mathrm{H}$-like mother ion. By definition of the mass eigenstates, the mass eigenstates of the H-like mother ion $\left|m^{\prime}\right\rangle$ and $\left|m^{\prime \prime}\right\rangle$ should be orthogonal $\left\langle m^{\prime} \mid m^{\prime \prime}\right\rangle=0$.

\section{EC- and $\beta^{+}$-Decay Rates, Caused by the Doubling of the Ground State of the Nuclei}

The EC-decay rate of the mother ion from the $|m\rangle$ state is equal to [3]

$$
\lambda_{\mathrm{EC}}^{(m)}(t)=\lambda_{\mathrm{EC}}\left(1+\sin 2 \theta \cos \left(\Delta E_{m^{\prime} m^{\prime \prime}} t\right)\right),
$$

where $\lambda_{\mathrm{EC}}$ is the EC-decay constant $[2,3,10]$ and $\Delta E_{m^{\prime} m^{\prime \prime}}$ is the energy difference of the ground mass eigenstates $\left|m^{\prime}\right\rangle$ and $\left|m^{\prime \prime}\right\rangle$. This shows a periodic dependence of the EC-decay rate with a period inversely proportional to $\Delta E_{m^{\prime} m^{\prime \prime}}$

$$
T_{\mathrm{EC}}=\frac{2 \pi \gamma}{\Delta E_{m^{\prime} m^{\prime \prime}}}
$$

For $T_{\mathrm{EC}}=7.06(8)$ seconds this gives $\Delta E_{m^{\prime} m^{\prime \prime}}=8.38(9) \times$ $10^{-16} \mathrm{eV}$. According to the experimental data [1], the amplitude of the time modulated term is equal to $a_{\mathrm{EC}} \simeq 0.20$. Since $a_{\mathrm{EC}}=\sin 2 \theta$, this gives $\theta \simeq 5.8^{0}$.

However, the H-like heavy ions, subjected to the ECdecays, are unstable also under $\beta^{+}$-decays [1]: $m \rightarrow d+e^{+}+$ $\nu_{e}$. Following the standard procedure for the calculation of the $\beta^{+}$-decay rates $[3,4,10]$ one gets

$$
\lambda_{\beta^{+}}^{(m)}(t)=\lambda_{\beta^{+}}\left(1+\sin 2 \theta \cos \left(\Delta E_{m^{\prime} m^{\prime \prime}} t\right)\right),
$$

where the $\beta^{+}$-decay constant $\lambda_{\beta^{+}}$has been calculated in [10]. Hence, according to $[8,9]$, the $\beta^{+}$-decay rates of the H-like heavy ions should have the same periodic time dependence as the EC-decay rates. This contradicts the experimental data on the time dependence of the $\beta^{+}$-decay rates of the $\mathrm{H}$ like heavy ${ }^{142} \mathrm{Pm}^{60+}$ ions at GSI [11-13], which indicate no time modulation. Of course, these experimental data are preliminary and one can wait for either the confirmation or rejection of them.

Nevertheless, this does not take away all problems. The point is that it seems that the doubling of the ground state of the nuclei of the $\mathrm{H}$-like heavy ions, proposed in $[8,9]$, is unable to generate time dependence of both EC-decay rates and $\beta^{+}$-decay rates of the $\mathrm{H}$-like heavy ions at all. Indeed, the ground mass eigenstates $\left|m^{\prime}\right\rangle$ and $\left|m^{\prime \prime}\right\rangle$ of the mother H-like heavy ions, injected into the Experimental Storage Ring (ESR), should be statistically populated by the fast projectile fragmentation (see (11) and discussions below). Such a process populates also statistically the system of the mother H-like heavy ions with coherent states $|\tilde{m}\rangle=-\sin \theta\left|m^{\prime}\right\rangle+\cos \theta\left|m^{\prime \prime}\right\rangle$. Due to statistical equivalence and indistinguishability of the coherent states $|m\rangle=\cos \theta\left|m^{\prime}\right\rangle+\sin \theta\left|m^{\prime \prime}\right\rangle$ and $|\tilde{m}\rangle=-\sin \theta\left|m^{\prime}\right\rangle+$ $\cos \theta\left|m^{\prime \prime}\right\rangle$ the probabilities $P_{m}$ and $P_{\tilde{m}}$ of the production of the coherent states $|m\rangle$ and $|\tilde{m}\rangle$, related by $P_{m}+P_{\tilde{m}}=1$, should be equal $P_{m}=P_{\tilde{m}}=1 / 2$.

The decay rates $\lambda_{\mathrm{EC}}^{(\tilde{m})}(t)$ and $\lambda_{\beta^{+}}^{(\tilde{m})}(t)$ of the EC and $\beta^{+}$ decays of the H-like heavy ions from the coherent state $|\tilde{m}\rangle$ are equal to

$$
\begin{aligned}
& \lambda_{\mathrm{EC}}^{(\tilde{m})}(t)=\lambda_{\mathrm{EC}}\left(1-\sin 2 \theta \cos \left(\Delta E_{m^{\prime} m^{\prime \prime}} t\right)\right), \\
& \lambda_{\beta^{+}}^{(\tilde{m})}(t)=\lambda_{\beta^{+}}\left(1-\sin 2 \theta \cos \left(\Delta E_{m^{\prime} m^{\prime \prime}} t\right)\right) .
\end{aligned}
$$

The total EC-decay and $\beta^{+}$-decay rates of the H-like heavy ions from the coherent states $|m\rangle$ and $|\tilde{m}\rangle$ are defined by

$$
\begin{aligned}
\lambda_{\mathrm{EC}}(t) & =P_{m} \lambda_{\mathrm{EC}}^{(m)}(t)+P_{\tilde{m}} \lambda_{\mathrm{EC}}^{(\tilde{m})}(t) \\
& =\lambda_{\mathrm{EC}}\left(1+\sin 2 \theta\left(P_{m}-P_{\tilde{m}}\right) \cos \left(\Delta E_{m^{\prime} m^{\prime \prime}} t\right)\right) \\
& =\lambda_{\mathrm{EC}}, \\
\lambda_{\beta^{+}}(t) & =P_{m} \lambda_{\beta^{+}}^{(m)}(t)+P_{\tilde{m}} \lambda_{\beta^{+}}^{(\tilde{m})}(t) \\
& =\lambda_{\beta^{+}}\left(1+\sin 2 \theta\left(P_{m}-P_{\tilde{m}}\right) \cos \left(\Delta E_{m^{\prime} m^{\prime \prime}} t\right)\right) \\
& =\lambda_{\beta^{+}} .
\end{aligned}
$$


Since $P_{m}=P_{\widetilde{m}}=1 / 2$, no interference terms and time dependence appear in the EC-decay and $\beta^{+}$-decay rates of the $\mathrm{H}$-like heavy ions.

This implies that the mechanism of two closely spaced ground mass eigenstates of the nuclei of the mother $\mathrm{H}$ like heavy ions is unable to provide a correct simultaneous description of the EC-decay and $\beta^{+}$-decay rates of the H-like heavy ions, measured at GSI $[1,11-13]$.

\section{Dynamics of Statistical Population}

What is the dynamics of a statistical population of the ground mass eigenstates $\left|m^{\prime}\right\rangle$ and $\left|m^{\prime \prime}\right\rangle$ and, correspondingly, the coherent states $|m\rangle$ and $|\tilde{m}\rangle$ of the mother H-like heavy ions in experiments at GSI?

At the GSI experiments the H-like heavy ions ${ }^{A} X^{(Z-1)+}$ are produced in the reaction [11-13]

$$
{ }^{152} \mathrm{Sm}+{ }^{9} \mathrm{~B} \mathrm{e} \longrightarrow{ }^{A} X^{(Z-1)+}+\cdots,
$$

where the incident ions ${ }^{152} \mathrm{Sm}$ with $500-600 \mathrm{MeV}$ kinetic energy per nucleon produce on a beryllium target ${ }^{9} \mathrm{Be}$ the fragments of the highly ionised states ${ }^{A} X^{(Z-1)+}$ like the $\mathrm{H}$-like ions ${ }^{140} \mathrm{Pr}^{58+},{ }^{142} \mathrm{Pr}^{60+},{ }^{122} \mathrm{I}^{52+}$, and so on, which are injected then with a kinetic energy of $400 \mathrm{MeV}$ per nucleon into the ESR [11-13].

According to the theory of high-energy nucleus-nucleus (or ion-ion) collisions [14], in the reactions (11) heavy nuclei ${ }^{A} X^{* Z+}$ are produced in excited states with excitation energies $E^{*}$. The excited energy levels of the nucleus ${ }^{A} X^{* Z+}$ are distributed statistically with an energy level density $\rho\left(E^{*}\right)$. According to the theory of nuclear energy level density and the Bethe theorem [14-22], a nuclear energy level density $\rho\left(E^{*}\right)$ is a continuous function of $E^{*}$, which can be deduced from a statistical analysis. The Bethe theorem gives the following general expression for the nuclear energy level density $\rho\left(E^{*}\right)[14-22]$

$$
\rho\left(E^{*}\right) \sim e^{S\left[E^{*}, T\right]},
$$

where $T$ has the meaning of nuclear temperature and $S\left[E^{*}, T\right]$ is the entropy of the Fermi system ${ }^{A} X^{* Z+}$ of nucleons with a given number $A$ [14-22].

Let, following $[8,9]$, the ground state of heavy nucleus ${ }^{A} X^{Z+}$ be doubled with masses $M_{1}$ and $M_{2}$ and the masssplitting $\left|M_{1}-M_{2}\right| \sim 10^{-15} \mathrm{eV}$. According to [14-22], a transition of the nucleus ${ }^{A} X^{* Z+}$ from the excited states with a nuclear energy level distribution $\rho\left(E^{*}\right)$ to the less excited states and finally to the ground states of the nucleus ${ }^{A} X^{Z+}$ with quantum numbers $J^{\pi}=1^{+}[10]$ should have a statistical character [18]. As a result the ground states of the nucleus ${ }^{A} X^{Z+}$ with quantum numbers $J^{\pi}=1^{+}$and masses $M_{1}$ and $M_{2}$, produced in the reaction (11), are populated statistically.

A statistical population of the ground states of the nucleus ${ }^{A} X^{Z+}$ entails a statistical population of the mass eigenstates $\left|m^{\prime}\right\rangle$ and $\left|m^{\prime \prime}\right\rangle$ of the H-like ion ${ }^{A} X^{(Z-1)+}$ with masses $M_{m^{\prime}}$ and $M_{m^{\prime \prime}}$ and the mass-difference $\mid M_{m^{\prime}}-$ $M_{m^{\prime \prime \prime}} \mid \sim 10^{-15} \mathrm{eV}$, produced in the reaction (11). As a result coherent states $|m\rangle=\cos \theta\left|m^{\prime}\right\rangle+\sin \theta\left|m^{\prime \prime}\right\rangle$ and $|\tilde{m}\rangle=-\sin \theta\left|m^{\prime}\right\rangle+\cos \theta\left|m^{\prime \prime}\right\rangle$ should be created with equal probabilities $P_{m}=P_{\widetilde{m}}=1 / 2$, that prohibits any time dependence of both the total EC-decay rates and the $\beta^{+}$decay rates of $\mathrm{H}$-like heavy ${ }^{A} \mathrm{X}^{(Z-1)+}$ ions.

We would like to notice that in reaction (11) the H-like ions ${ }^{A} X^{(Z-1)+}$ are produced both in the ground hyperfine states with atomic spin $F=1 / 2$ and in the excited hyperfine state with atomic spin $F=3 / 2$, which decays into the ground hyperfine state ${ }^{A} X_{F=3 / 2}^{(Z-1)+} \rightarrow{ }^{A} X_{F=1 / 2}^{(Z-1)+}+\gamma$ with the lifetime of order of $\tau \sim 10^{-2} \mathrm{~s}[10]$. Of course, such transitions should replenish statistically the system of the mother $\mathrm{H}$-like heavy ions with the ground hyperfine states ${ }^{A} X_{F=1 / 2}^{(Z-1)+}$ with masses $M_{m^{\prime}}$ and $M_{m^{\prime \prime}}$ and a mass splitting $\left|M_{m^{\prime}}-M_{m^{\prime \prime}}\right| \sim 10^{-15} \mathrm{eV}$.

\section{Conclusion}

We have analysed the mechanism of two closely space mass eigenstates of the $\mathrm{H}$-like heavy ions with a mass splitting of order of $10^{-15} \mathrm{eV}$. We have applied this mechanism to the calculation of the time modulation of the $\beta^{+}$-decay rates of the $\mathrm{H}$-like heavy ions and analysed the dynamics of the production of the $\mathrm{H}$-like heavy ions at GSI experiments.

We have shown that in case the nuclei of the H-like heavy ions have the ground states splitted with a massdifference of order of $10^{-15} \mathrm{eV}$, the $\beta^{+}$-decay rates of the $\mathrm{H}$-like heavy ions, decaying from the coherent state $|m\rangle=$ $\cos \theta\left|m^{\prime}\right\rangle+\sin \theta\left|m^{\prime \prime}\right\rangle$, should have the same period of the time dependence as the EC-decay rates. This contradicts recent experimental data at GSI [11-13].

We have analysed the dynamics of the production of the $\mathrm{H}$-like heavy ions with two closely spaced mass eigenstates at GSI experiments. We have shown that, according to the theory of high-energy ion-ion collisions [14-22], the system of the H-like heavy ions, injected into the ESR with an kinetic energy of about $400 \mathrm{MeV}$ per nucleon, should be statistically populated with two closely spaced ground mass eigenstates $\left|m^{\prime}\right\rangle$ and $\left|m^{\prime \prime}\right\rangle$. Of course, the statistical population of the states $\left|m^{\prime}\right\rangle$ and $\left|m^{\prime \prime}\right\rangle$ is not only determined by the energy-level density of the states in the nucleus produced in reaction (11), but also by $\gamma$ transitions, defined by the $\gamma$-strength functions [23], that lead to de-excitation of the excited states populated directly in the nuclear reactions. Since such a statistical population of the nuclear states leads to a statistical equivalence of both two closely spaced ground mass eigenstates $\left|m^{\prime}\right\rangle$ and $\left|m^{\prime \prime}\right\rangle$ and their coherent superpositions $|m\rangle$ and $|\tilde{m}\rangle$, the EC and $\beta^{+}$decay rates of the $\mathrm{H}$-like heavy ions do not depend on time at all. Thus, we can conclude that such a hypothesis of two closely spaced ground mass eigenstates of heavy nuclei is unable to explain correctly the experimental data on the time modulation of both the EC-decay rates and $\beta^{+}$-decay rates, measured at GSI $[1,11-13]$.

As we have mentioned above the mass splitting of the $\mathrm{H}$ like mother ion can be attributed to the splitting of the energy level of the bound electron. However, since in this case the coherent state $|m\rangle$, normalised to unity, reduces to the wave function of the unperturbed state of the $\mathrm{H}$-like mother ion with a common time dependent phase, the splitting of the 
energy level of the bound electron of the H-like mother ion leads to no time modulation for the EC and the $\beta^{+}$decay rates the H-like mother ion.

\section{Acknowledgment}

The authors acknowledge fruitful discussions with T. Ericson.

\section{References}

[1] Y. A. Litvinov, F. Bosch, N. Winckler, et al., "Observation of non-exponential orbital electron capture decays of hydrogenlike ${ }^{140} \mathrm{Pr}$ and ${ }^{142} \mathrm{Pm}$ ions," Physics Letters B, vol. 664, no. 3, pp. 162-168, 2008.

[2] A. N. Ivanov and P. Kienle, "Time modulation of the $K$ shell electron capture decay rates of $\mathrm{H}$-like heavy ions at GSI experiments," Physical Review Letters, vol. 103, no. 6, Article ID 062502, 4 pages, 2009.

[3] A. N. Ivanov, E. L. Kryshen, M. Pitschmann, and P. Kienle, "Comments on "Rates of processes with coherent production of different particles and the GSI time anomaly", http://arxiv.org/abs/0807.2750.

[4] A. N. Ivanov, E. L. Kryshen, M. Pitschmann, and P. Kienle, "Time modulation of the $\beta^{+}$-decay rate of $\mathrm{H}$-like ${ }^{140} \mathrm{Pr}^{58+}$ ions," Physical Review Letters, vol. 1, no. 18, Article ID 182501, 4 pages, 2008.

[5] H. J. Andrä, "Fine structure, hyperfine structure and lamb shift measurements by the beam-foil technique," Physica Scripta, vol. 9, pp. 257-280, 1974.

[6] H. J. Andrä, "Stark-induced quantum beats in $\operatorname{HLY}_{\alpha}$ emission," Physical Review A, vol. 2, no. 6, pp. 2200-2207, 1970.

[7] W. W. Chow, M. O. Scully, and J. O. Stoner Jr., "Quantumbeat phenomena described by quantum electrodynamics and neoclassical theory," Physical Review A, vol. 11, no. 4, pp. 1380-1388, 1975.

[8] C. Giunti, "Rates of processes with coherent production of different particles and the GSI time anomaly," Physics Letters $B$, vol. 665, no. 2-3, pp. 92-94, 2008.

[9] H. Kienert, J. Kopp, M. Lindner, and A. Merle, "The GSI anomaly," Journal of Physics: Conference Series, vol. 136, Article ID 022049, 7 pages, 2008.

[10] A. N. Ivanov, M. Faber, R. Reda, and P. Kienle, "Weak decays of H-like ${ }^{140} \mathrm{Pr}^{58+}$ and He-like ${ }^{140} \mathrm{Pr}^{57+}$ ions," Physical Review C, vol. 78, no. 2, Article ID 025503, 4 pages, 2008.

[11] P. Kienle, "Time-modulation of orbital electron capture decays by mixing of massive neutrinos," Nuclear Physics A, vol. 827, no. 1-4, pp. 510c-517c, 2009.

[12] P. Kienle, "Two-body weak decay studies in an ion storage ring," Journal of Physics: Conference Series, vol. 171, Article ID 012065, 6 pages, 2009.

[13] N. Winckler, et al., "GSI Annual report,” Tech. Rep., 2008.

[14] V. S. Barashenkov and B. D. Toneev, High-Energy Interactions of Particles and Atomic Nuclei with Nuclei, Atomizdat, Moscow, Russia, 1972.

[15] H. A. Bethe, "An attempt to calculate the number of energy levels of a heavy nucleus," Physical Review, vol. 50, no. 7, pp. 332-341, 1936.

[16] H. A. Bethe, "Nuclear physics B. Nuclear dynamics, theoretical," Reviews of Modern Physics, vol. 9, no. 2, pp. 69-244, 1937.
[17] C. van Lier and G. E. Uhlenbeck, "On the statistical calculation of the density of the energy levels of the nuclei," Physica, vol. 4, no. 7, pp. 531-542, 1937.

[18] V. Weisskopf, "Statistics and nuclear reactions," Physical Review, vol. 52, no. 4, pp. 295-303, 1937.

[19] N. Rosenzweig, "Level density of a system of fermi particles," Physical Review, vol. 105, no. 3, pp. 950-956, 1957.

[20] T. Ericson, "On the level density of deformed nuclei," Nuclear Physicsw, vol. 6, pp. 62-81, 1958.

[21] K. J. Le Couteur and D. W. Lang, "Neutron evaporation and level densities in excited nuclei," Nuclear Physics, vol. 13, no. 1, pp. 32-52, 1959.

[22] N. A. Bethe and P. Morrison, Elementary Nuclear Theory, John Wiley \& Sons, New York, NY, USA, 1961.

[23] A. Schiller, L. Bergholt, M. Guttormsen, E. Melby, J. Rekstad, and S. Siem, "Extraction of level density and $\gamma$ strength function from primary $\gamma$ spectra," Nuclear Instruments and Methods in Physics Research Section A, vol. 447, no. 3, pp. 498$511,2000$. 

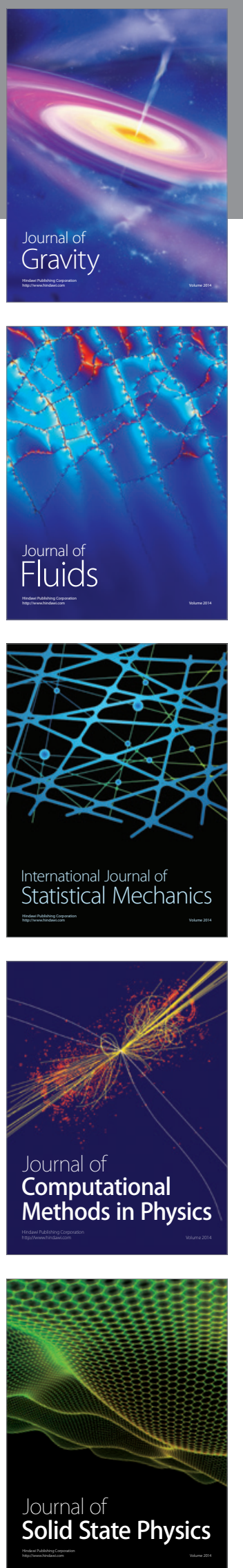

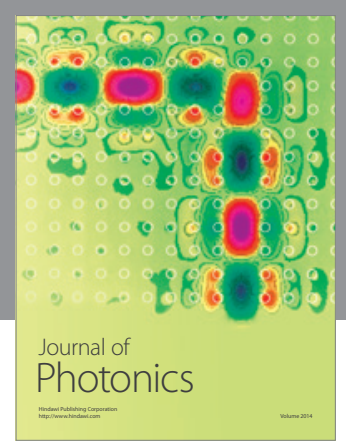

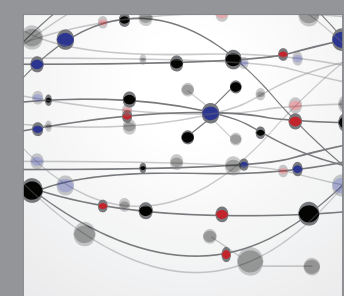

The Scientific World Journal
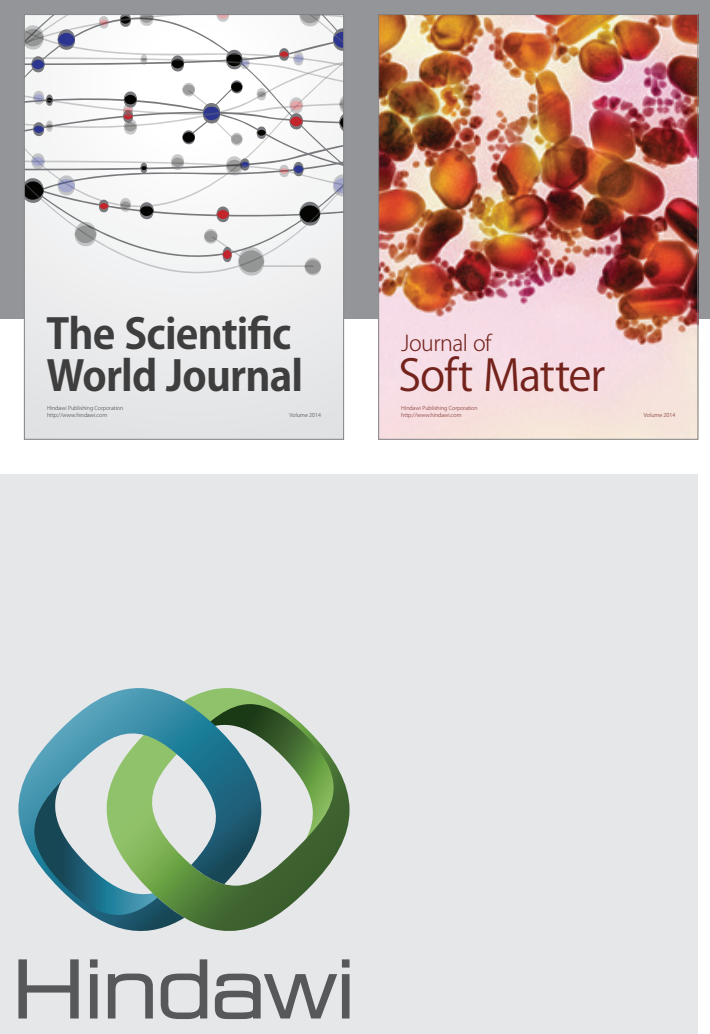

Submit your manuscripts at

http://www.hindawi.com
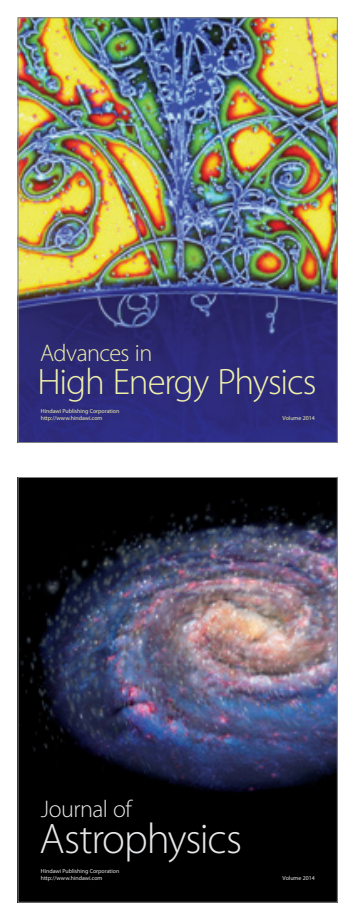
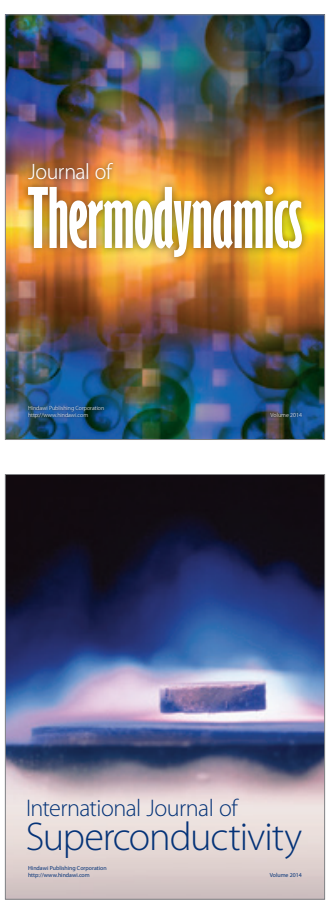
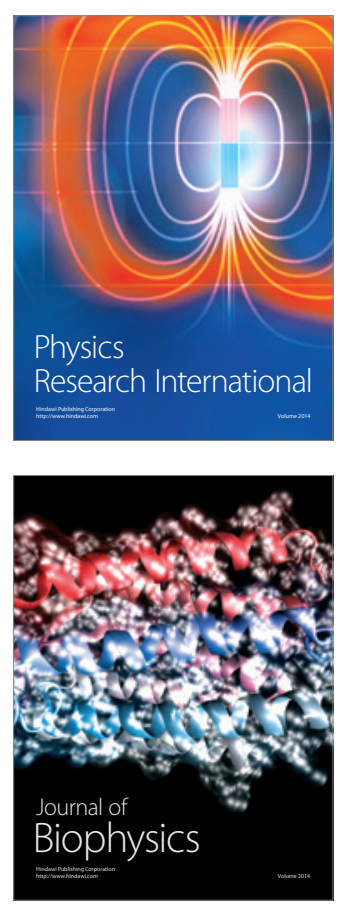
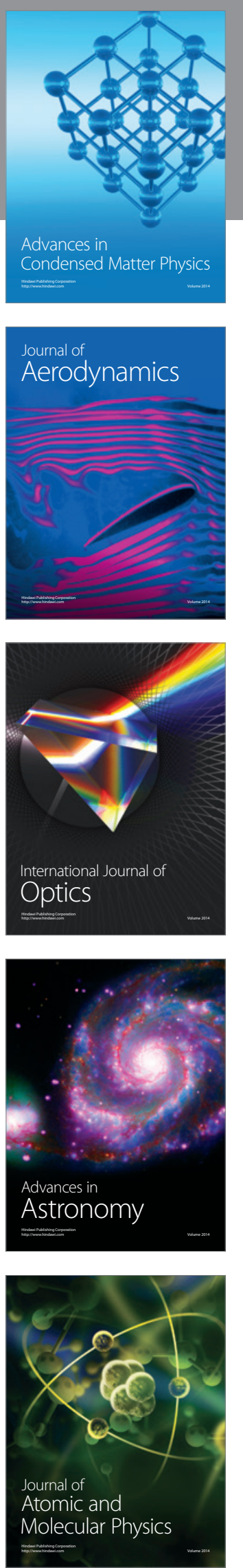